\title{
Lentiviral-mediated RNA interference targeting stathmin 1 gene in human gastric cancer cells inhibits proliferation in vitro and tumor growth in vivo
}

Javed Akhtar, Zhou Wang ${ }^{*}$, Zhi Ping Zhang and Ming Ming Bi

\begin{abstract}
Background: Gastric cancer is highly aggressive disease. Despite advances in diagnosis and therapy, the prognosis is still poor. Various genetic and molecular alterations are found in gastric cancer that underlies the malignant transformation of gastric mucosa during the multistep process of gastric cancer pathogenesis. The detailed mechanism of the gastric cancer development remains uncertain. In present study we investigated the potential role of stathmin 1 gene in gastric cancer tumorigenesis and examined the usefulness of RNA interference (RNAi) targeting stathmin1 as a form of gastric cancer treatment.
\end{abstract}

Methods: A lentiviral vector encoding a short hairpin RNA (shRNA) targeted against stathmin1 was constructed and transfected into the packaging cells HEK 293 T and the viral supernatant was collected to transfect MKN-45 cells. The transwell chemotaxis assay and the CCK-8 assay were used to measure migration and proliferation of tumor cells, respectively. Quantitative real-time PCR and western blotting were used to detect the expression levels of stathmin 1.

Results: Lentivirus mediated RNAi effectively reduced stathmin1 expression in gastric cells. Significant decreases in stathmin 1 mRNA and protein expression were detected in gastric cells carrying lentiviral stathmin-shRNA vector and also significantly inhibited the proliferation, migration in gastric cancer cells and tumorigenicity in Xenograft Animal Models.

Conclusions: Our findings suggest that stathmin1 overexpression is common in gastric cancer and may play a role in its pathogenesis. Lentivirus mediated RNAi effectively reduced stathmin1 expression in gastric cells. In summary, shRNA targeting of stathmin1 can effectively inhibits human gastric cancer cell growth in vivo and may be a potential therapeutic strategy for gastric cancer.

Keywords: Stathmin1, Gastric cancer, Lentivirus, shRNA

\section{Background}

Gastric cancer is one of the most common cancers worldwide with approximately 989,600 new cases and 738,000 deaths per year, accounting for about 8 percent of new cancers [1]. The worldwide incidence of gastric cancer has declined rapidly over the recent few decades. Despite its recent decline, gastric cancer is the fourth

\footnotetext{
* Correspondence: wz620226@hotmail.com

Department of Thoracic Surgery, Provincial Hospital Affiliated to Shandong University, 250021, Shandong, China
}

\section{() Biomed Central}

(c) 2013 Akhtar et al.; licensee BioMed Central Ltd. This is an Open Access article distributed under the terms of the Creative Commons Attribution License (http://creativecommons.org/licenses/by/2.0), which permits unrestricted use, distribution, and reproduction in any medium, provided the original work is properly cited.

most common cancer and the second leading cause of cancer-related death worldwide [2,3]. It has the highest incidence in China, Japan, Iran, Korea and eastern Asia. The overall prognosis is poor with a 5-year survival rate below $30 \%$ in most countries [4]. In China, the decline was less dramatic than other countries; despite an overall decrease in gastric cancer incidence, an increase has been observed in the oldest and the youngest group, and a less remarkable decline has been observed among women than in men [5]. Unraveling the molecular mechanisms underlying gastric carcinogenesis is one of 
the major challenges in cancer genomics. Malignant transformation of gastric cells is the consequence of a multistep process involving different genetic and epigenetic changes in numerous genes in combination with host genetic background and environmental factors [6].

Stathmin1/oncoprotein 18, also known as STMN-1, is a highly conserved $17 \mathrm{kDa}$ protein. Its function as an important regulatory protein of microtubule dynamics has been well characterized [7]. The protein is conserved in vertebrates and expressed in most tissues [7]. Stathmin performs an important function in regulating rapid microtubule remodeling of the cytoskeleton in response to the cell's needs. Stathmin's role in regulation of the cell cycle causes it to be an oncoprotein named oncoprotein 18 (op18). Stathmin can cause uncontrolled cell proliferation when mutated and not functioning properly. Stathmin 1 is one of the microtubule-regulating proteins that play a critical role in the assembly and disassembly of the mitotic spindle [8-11]. It is highly expressed in a wide variety of human cancers, including leukemia, breast, prostate, and lung cancer, and provides an attractive target for cancer therapy [12-15]. Our study on esophageal squamous cell cancer revealed that stathmin1 is a predictor of survival in stage IIA esophageal squamous cell carcinoma after surgery [16].

RNA interference (RNAi) is a post-transcriptional process triggered by the introduction of double-stranded RNA (dsRNA) which leads to gene silencing in a sequencespecific manner. The first evidence that dsRNA could achieve efficient gene silencing through RNAi came from studies on the nematode Caenorhabditis elegans. Further analyses in the fruit fly Drosophila melanogaster have contributed greatly toward understanding the biochemical nature of the RNAi pathway [17].

In this study, we have examined the effect of lentiviral mediated knockdown of stathmin gene expression in gastric cancer cells. We showed stathmin1 is overexpressed in gastric cancer derived cell lines MKN-45 and targeting its expression cause decrease in proliferation and migration of MKN-45 cell in vitro and tumor growth in vivo. At present, it is unclear whether stathmin 1 is associated with gastric carcinogenesis. The major purpose of this study was therefore to examine the expression of stathmin 1 protein and mRNA in gastric cancer-derived cell lines.

\section{Methods}

\section{Mice, cell lines, culture medium and reagents}

BALB/C-nu/nu male mice (weight 18-22 g, 6 weeks old), Human gastric carcinoma cell line MKN-45, Human Embryonic Kidney 293 cell (HEK93T) were obtained from the Shanghai Tumor Institution. Classical Liquid Media Dulbecco's Modified Eagles Medium (DMEM), High Glucose RPMI Media 1640 was purchased from HyClone (Thermo Scientific). Fetal bovine serum (FBS) was purchased from Gibco (Invitrogen Co., USA). Dimethyl sulfoxide (DMSO) was purchased from SigmaAldrich (St Louis, Missouri, USA). Rabbit Anti-STMN-1 Polyclonal antibody and HRP-conjugated secondary antibodies were purchased from Abcam (Cambridge, MA).

All experimental procedures using animals in the present study had received prior approval by the Institutional Animal Care and Use Committee of Shandong University under Contract 2011-0015.

\section{Cell culture}

MKN-45 cell lines were grown in RPMI 1640 medium supplemented with $10 \%$ fetal bovine serum (FBS) and 100 units $/ \mathrm{ml}$ penicillin, and $100 \mathrm{mg} / \mathrm{ml}$ streptomycin (Gibco). The cells were grown at $37^{\circ} \mathrm{C}$ in a humidified atmosphere containing 5\% CO2. Stock cultures of each cell line were routinely sub-cultured at least once a week and the medium was changed every $2-3$ days.

\section{Lentivirus-mediated short hairpin RNA (shRNA) knockdown of gene expression}

pGIPZ-lentiviral shRNAmir vectors targeting human stathmin1 gene and Non-silencing pGIPZ control vector were purchased from Open Biosystems (Thermo Fisher Scientific, Inc.). pGIPZ non-silencing control vector for use as expression control to generate non-silencing lentiviral stock to optimize expression conditions in the mammalian cell line of interest. pGIPZ cloning vector containing Turbo GFP reporter and also elements required to allow packaging of the expression construct into virions (i.e. $5^{\prime}$ and $3^{\prime}$ LTRs and $\Psi$ packaging signal). pGIPZ vector also expresses a puromycin-resistant gene.

The sequences of STMN1 shRNA are as following: 5'-TTATTAGCTTCCATTTTGT-3' and 5'-TTATTAACC ATTCAAGTCC-3'. Lentiviral shRNA was produced by Co-transfection of the Trans-Lentiviral packaging mix with a shRNA transfer vector into HEK 293T packaging cells (OpenBiosystems). For cell infection, viral supernatants were supplemented with $6 \mu \mathrm{g} / \mathrm{mL}$ polybrene and incubated with cells for 24 hours. MKN-45 cells were transduced by the lentiviral particles followed by puromycin selection $(1 \mu \mathrm{g} / \mathrm{mL})$ for 10 days. The cells stably expressing shRNA were maintained in puromycin $(0.2 \mu \mathrm{g} / \mathrm{mL})$.

\section{RNA extraction and qRT-PCR}

Total RNA extraction was performed using Trizol reagent (Invitrogen) according to the manufacturer's instruction. RNA concentration was measured by Nano Drop 1000 (Thermo Fisher Scientific). One microgram of total RNA extracted from the cells was subjected to reverse Transcription (RT). Verso cDNA Ki (Thermo Scientific) was used for cDNA synthesis. Real-time RTPCR was used to quantify the expression level of Stmn1 
gene in gastric cancer cell lines MKN-45 using ABI 7300 real-time PCR thermal cycle instrument (ABI, USA), according to the supplied protocol. Amplification conditions were as follows: Reverse-transcription reaction: $42^{\circ} \mathrm{C}$, 30 minutes per cycle. PCR cycling conditions were as follows: Enzyme activation $95^{\circ} \mathrm{C} 15$ minutes per cycle, denaturation $95^{\circ} \mathrm{C}$ at 15 seconds per 40 cycles and Annealing/Extension at $60^{\circ} \mathrm{C}$ for 60 seconds.

A Real-time PCR reaction was performed using the Solaris qPCR Gene Expression Master Mix with LOW ROX premixed and $1 \mu \mathrm{L}$ of total cDNA in each well, Stathmin specific primers were as follows: (F, AGAATA CACTGCCTGTCGCTTG; R, AGGCACGCTTCTCCAG $T T)$. The relative expression levels were normalized to expression of endogenous Beta-Actin. Primers: (F, TGGA GAAAATCTGGCACCAC; R, GGTCTCAAACATGATCTGG).

\section{Protein extraction and Western blotting}

For whole-cell protein extraction, cells were washed with cold PBS and subsequently lysed in cold RIPA lysis buffer $(50 \mathrm{mM}$ Tris- $\mathrm{HCl}, \mathrm{pH}$ 7.4, $150 \mathrm{mM} \mathrm{NaCl}, 1 \mathrm{mM}$ dithiothreitol [DTT], 0.25\% sodium deoxycholate, $0.1 \%$ NP-40) containing $1 \mathrm{mM}$ phenylmethysulfonyl fluoride (PMSF), $50 \mathrm{mM}$ sodiumpyrophosphate, $1 \mathrm{mM} \mathrm{Na3VO4,}$ $1 \mathrm{mM} \mathrm{NaF}, 5 \mathrm{mM}$ EDTA, $5 \mathrm{mM}$ EGTA, and protease inhibitors cocktail (Roche). Cell lysis was performed on ice for 30 minutes. Clear protein extracts were obtained by centrifugation for 30 minutes at $4^{\circ} \mathrm{C}$. Protein concentrations were determined by the method of Bradford using the Bio-Rad protein assay reagent (Bio-Rad) and 20-40 $\mathrm{mg}$ of protein mixed with loading buffer was loaded per lane, separated by $12 \%$ SDS-polyacrylamide gel electrophoresis (SDS-PAGE). Proteins were transferred to PVDF membrane filters (Millipore, USA). Nonspecific binding was blocked by incubation in phosphate-buffered saline (PBS) containing 0.1\% Tween 20 (PBS-T) and 5\% skim milk. PVDF membranes were blocked with 5\% dry milk for one hour at $4^{\circ} \mathrm{C}$. Membranes were incubated in STMN1 primary antibody (1:1000) overnight at $4^{\circ} \mathrm{C}$. The membranes were then incubated with the corresponding secondary antibody (1:2000, horseradish peroxidase-conjugated anti-rabbit) in TBST-5\% nonfat milk for 1 hour at room temperature and the immunoreactive bands were visualized using EZ ECL Chemiluminescence Detection Kit for HRP (Biological Industries Ltd, Israel). Images were acquired using the LAS3000 Imager (Fujifilm). Membranes were re-probed for BetaActin as a loading control.

\section{Cell proliferation assay}

Cell Counting Kit-8 (CCK-8; Dojindo) was used in cell proliferation assay. 3000 viable cells per well into 96well tissue culture plates in a final volume of $100 \mu \mathrm{l}$. Every 24 hours, a plate was subjected to assay by adding
$10 \mu \mathrm{l}$ of CCK-8 solution to each well, and the plate was further incubated for 4 hours at $37^{\circ} \mathrm{C}$. The absorbance at $450 \mathrm{~nm}$ was measured with a micro plate reader. The experiment was performed in eight replicates.

\section{Migration and invasion assays}

For trans-well migration assay, 50,000 cells were added to upper chamber in serum free media and migration at $37^{\circ} \mathrm{C}$ towards $10 \%$ FBS containing growth media was determined either after 24 hours or 48 hrs. Cells migrated through the membrane were fixed, stained with H\&E (Sigma) and counted under light microscope. For invasion assay, lower chambers of matrigel coated invasion plates were coated with $10 \mathrm{mg} / \mathrm{ml}$ fibronectin overnight at $4^{\circ} \mathrm{C}$ and cells invading through matrigel were fixed and stained after 48 hours.

\section{Immunocytochemistry}

Stably transfected MKN-45 cells were seeded into 4chambered glass slides (Nunc Lab-Tek Chamber Slide System). Cells were then incubated overnight. After 24 hours, cells were rinsed with PBS, fixed with 3.7\% w/v paraformaldehyde (Sigma), rinsed with PBS, and permeablized in $0.5 \%$ Triton X-100 (Sigma). Nonspecific immunoglobulin binding was blocked with 5\% normal goat serum and 0.5\% NP-40 (Sigma). Primary antibodies recognizing with stathmin 1 (abicam) were diluted 1:100 in blocking solution. After incubation with primary antibody, cells were rinsed with $0.05 \%$ Tween-20 (Bio-Rad) in PBS, and then incubated with secondary antibody for $1 \mathrm{~h}$ at RT. Stain with 3,3'-Diaminobenzidine (DAB) and observed under light microscope.

\section{Immunofluorescence}

To examine the protein expression of stathmin 1 we performed immunofluorescence analysis. Briefly, the cells were washed with PBS and fixed in $4 \%$ paraformaldehyde for 10 minutes at $37^{\circ} \mathrm{C}$ followed by absolute methanol for 10 minutes at $4^{\circ} \mathrm{C}$ and blocked in PBS containing $1 \%$ skimmed milk for 10 minutes. The samples were then incubated with affinity-purified Rabbit Anti-Human Stathmin 1 Polyclonal Antibody (Abcam, USA) at a dilution of 1:100 for 2 hours, rinsed twice with PBS, and incubated with goat anti-rabbit IgG Alexa Fluor 488 (Molecular Probes) for 1 hour.

\section{In vivo studies of gastric cancer xenograft tumor models in nude mice}

Six-week-old male BALB/c nude mice were housed in a temperature-controlled, pathogen-free animal facility with 12-hour light and dark cycles. Mice were injected subcutaneously into bilateral flanks with untransfected cells, or transfected with Non-silencing shRNA and stathmin shRNA $\left(2 \times 10^{6}\right.$ cells in $200 \mu \mathrm{l}$ PBS $)$ to establish 
tumors. Tumor mass (xenograft) volume was measured every week from week 3 to week 7 . After 7 week mice were sacrificed, and tumors were harvested.

\section{Statistical analysis}

For comparison of more than three groups, we used one-way analysis of variance, followed by Tukey's multiple comparison-values $<0.05$ were considered statistically significant. One-way analysis of variance (ANOVA), followed by the LSD post hoc test was used to compare mean differences in 2 or more groups. All statistical analysis was performed by using IBM SPSS version 20.0.

\section{Results}

\section{Stahmin1/Op18 expression in MKN-45 cell lines}

We assessed the stathmin 1 gene expression in gastric cancer derived cell lines MKN-45 by immunofluorescence (IF) analysis as shown in the (Figure 1A, B) and immunocytochemistry (ICC) (Figure 1C, D). Strong immunoreactivity of stathmin 1 protein was detected in the cytoplasm of MKN-45 cells. We also evaluated the state of stathmin 1 protein expression in MKN-45 cell line by Western blot analysis (Figure 2A).

\section{Transfection \& transduction efficiency}

Successful packing of lentivirus was done in HEK 293 T. To determines the efficacies of viral vectors, viral supernatants prepared from either non-silencing-shRNA (control) or stathmin1shRNA were added to gastric cancer cells. Transfection efficiencies were determined by fluorescence microscopy. To evaluate the silencing efficiency, qRT-PCR and western blotting analysis were performed.

\section{Lentivirus-mediated RNAi efficiently suppressed}

\section{stathmin 1 protein and mRNA expression in MKN-45 cells}

Stable expression of stathmin1 shRNAs specifically knocks down the stathmin1 expression and activity in MKN-45 gastric cells. MKN-45 cells express high levels of stathmin1, and are aggressively growing and metastatic. To investigate the role of stathmin1 in MKN-45 cell's growth and metastasis, we constructed lentivirus vector with stathmin 1 shRNA and infected MKN-45 cells. After viral infection, more than $95 \%$ of the cells were GFP-positive, indicating a high efficiency of shRNA delivery. Stathmin1 shRNA2 more efficiently knockdown protein expression in MKN-45 cell line as compared to normal control and non-silencing group $(\mathrm{p}<0.05)$ (Figure 2A, B). Stathmin1 shRNA2 also efficiently suppressed the stathmin1 mRNA level conformed by

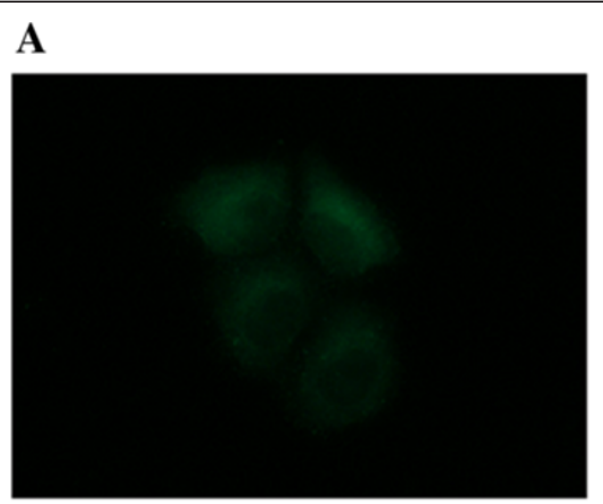

\section{B}

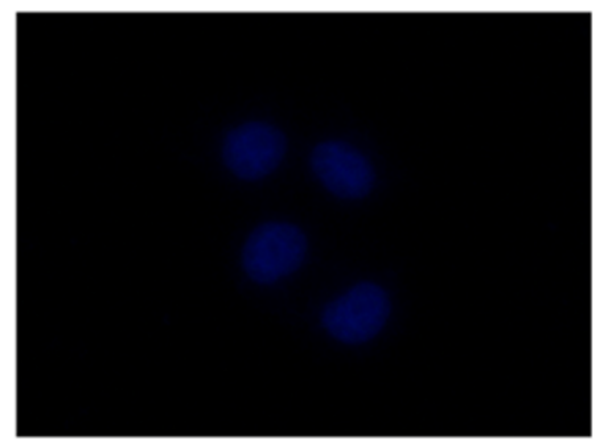

C

D
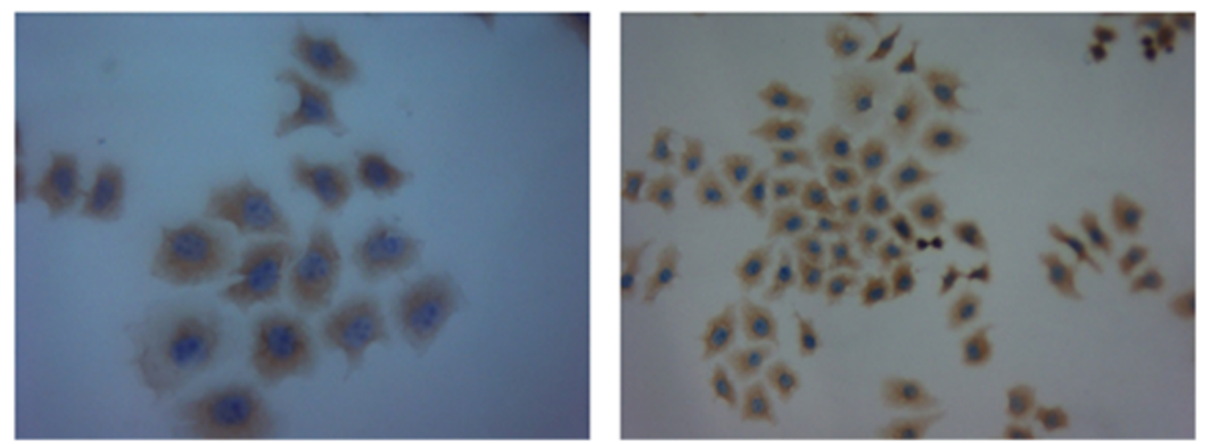

Figure 1 Expression of stathmin1 in human gastric cancer cells line MKN-45. (A) Immunofluorescences analysis of MKN-45 cells showing stathmin 1 overexpression in MKN-45 cells and, (B) DAPI stained cells (blue). (C) \& (D) Immunocytochemical analysis shows strong immunoreactivity of stathmin1 in the cytoplasm of MKN-45. The data are representative from three independent experiments with similar results. 
$\mathbf{A}$

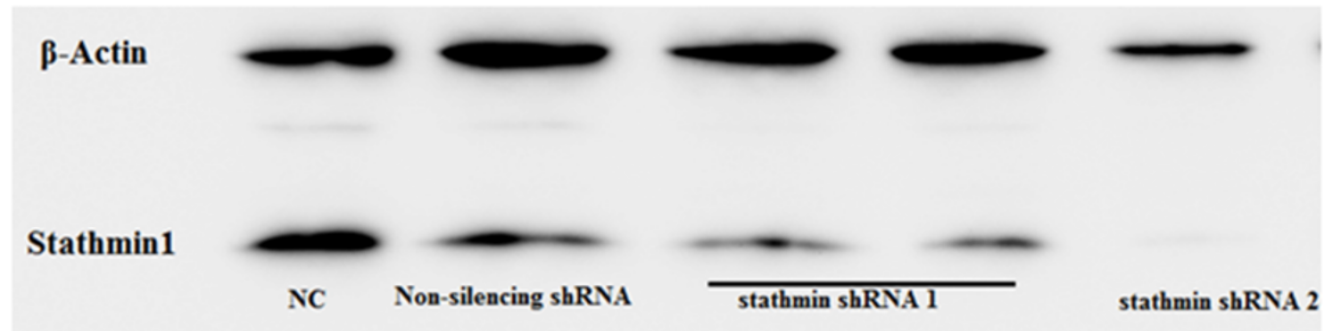

$\mathbf{B}$

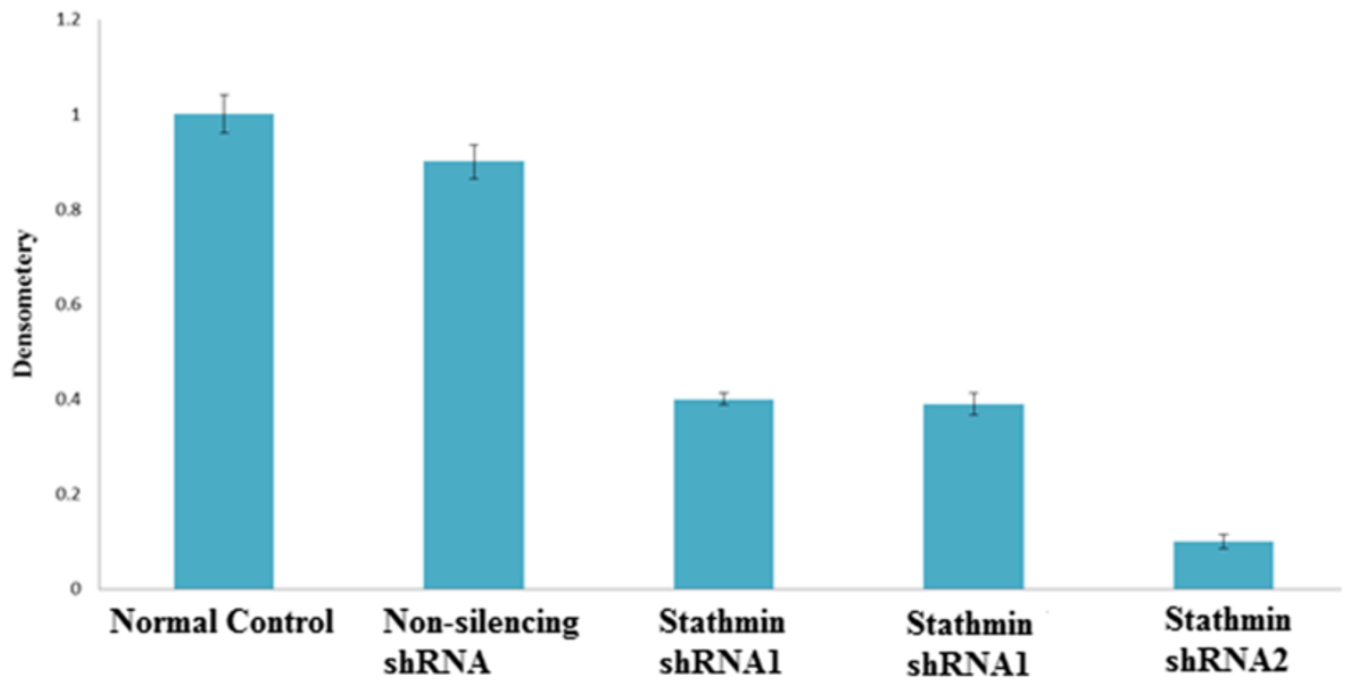

C qRT-PCR

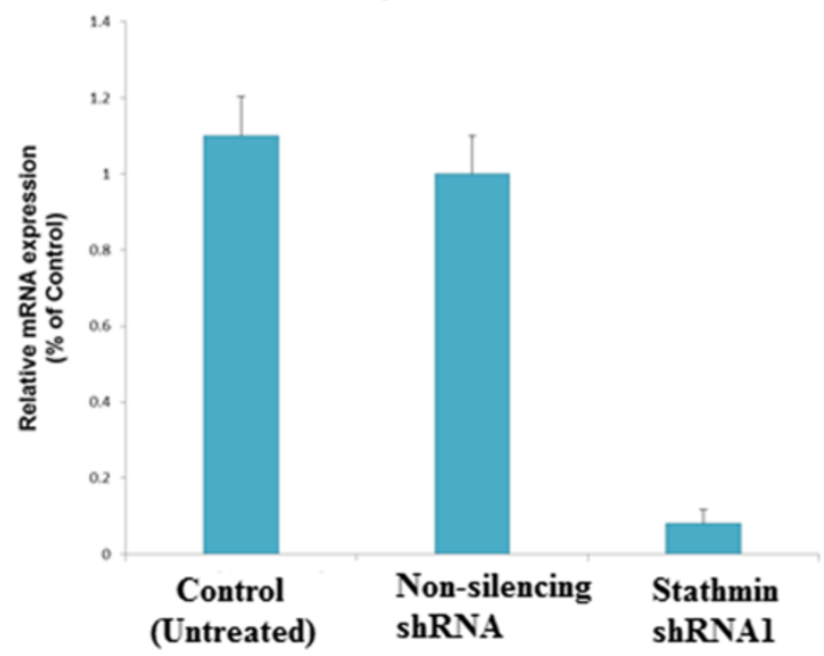

Figure 2 Western blot Assay and qRT-PCR analysis of gastric cancer-derived cell lines MKN-45 with Non-silencing shRNA and stathmin shRNA knockdown. (A) MKN-45 cells were transfected with two different Stathmin1-specific shRNA or with a Non-silencing shRNA. Forty-eight hours after transfection, the cells were analyzed by western blotting for stathmin1 (B) Densitometry analysis of Western blots from three independent experiments, respectively, showing that stathmin1 shRNA specifically knockdown stathmin1 expression in gastric cancer cell lines (C) Bar graph showing qRT-PCR analysis of cells transfected with the shRNA2 using primers specific for stathmin1 or $\beta$-actin mRNA. stathmin 1 shRNA2 significantly down-regulate stathmin mRNA level as compared with Control or transduction with Non-silenecing shRNA in MKN-45 cell lines. Data are expressed as percentage change (Means \pm S.D.) compared with controls and represent four independent experiments. $(P<0.05 \mathrm{vs}$ Non-silencing shRNA, one-way analysis of variance (ANOVA) followed by Tukey's multiple comparion). 
qRT-PCR. We selected stathmin1 shRNA2 for further study (Figure 2C).

Stable knockdown of oncogenic stathmin 1 by lenti-shRNA significantly inhibited gastric cell growth in vitro

Frequent up-regulation of stathmin 1 mRNA and protein in gastric cancer cell suggested a potential oncogenic role of this gene. Stathmin1 targeting impaired proliferation of MKN-45 cell lines. To investigate the possible antiproliferative effects of stathmin 1 knockdown in vitro, a CCK-8 assay was performed and a cell growth curve was generated. Stathmin1 shRNA2 transduced MKN-45 cells showed significantly reduced viability relative to control group. (Figure 3A). Stathmin1 knockdown inhibited the proliferation of MKN-45 cells in vitro, indicating that the expression of stathmin 1 affects the growth of gastric cells.

\section{Stable knockdown of stathmin 1 expression inhibits} invasion and migration of MKN-cells

To evaluate the function of stathmin1sh RNA on gastric cancer cell invasion, Matrigel invasion chambers were utilized. Inhibited stathmin1 expression led to a significantly decreased invasive ability of gastric cancer cells (Figure 3B).
Cell migration was evaluated in the Boyden migration assay two days after MKN-45 cells were transfected with stathmin 1 shRNA2 or transfected (control) group. Cells with motile capacity could migrate through the pores of the Trans well filters because of the attraction to $10 \%$ FBS in the lower chamber. Cells transfected with stathmin1 shRNA2 displayed less migration compared with non-transfected control cells, three-fold increases in the number of migrating cells (Figure $3 \mathrm{C}, \mathrm{D}, \mathrm{E}$ ). The introduction of stathmin1shRN into cells, however, markedly decreased the cell migration, in comparison to non-transfected cells.

\section{In vivo studies of gastric cancer xenograft tumor models in nude mice}

To further evaluate the effects of reduced stathmin 1 expression on the tumorigenic phenotype and in particular its contribution to in vivo tumor growth. MKN-45 cells infected with non-silencing- shRNA and stathmin1 shRNA2 or untreated were injected into mice, stathmin shRNA2 transfected grew rapidly as compared to negative control $(\mathrm{p}<0.05)$ (Figure 4A, B). These results demonstrate that in vivo tumor growth was inhibited by shRNA-mediated knockdown of stathmin1 expression in MKN-45 cell lines.

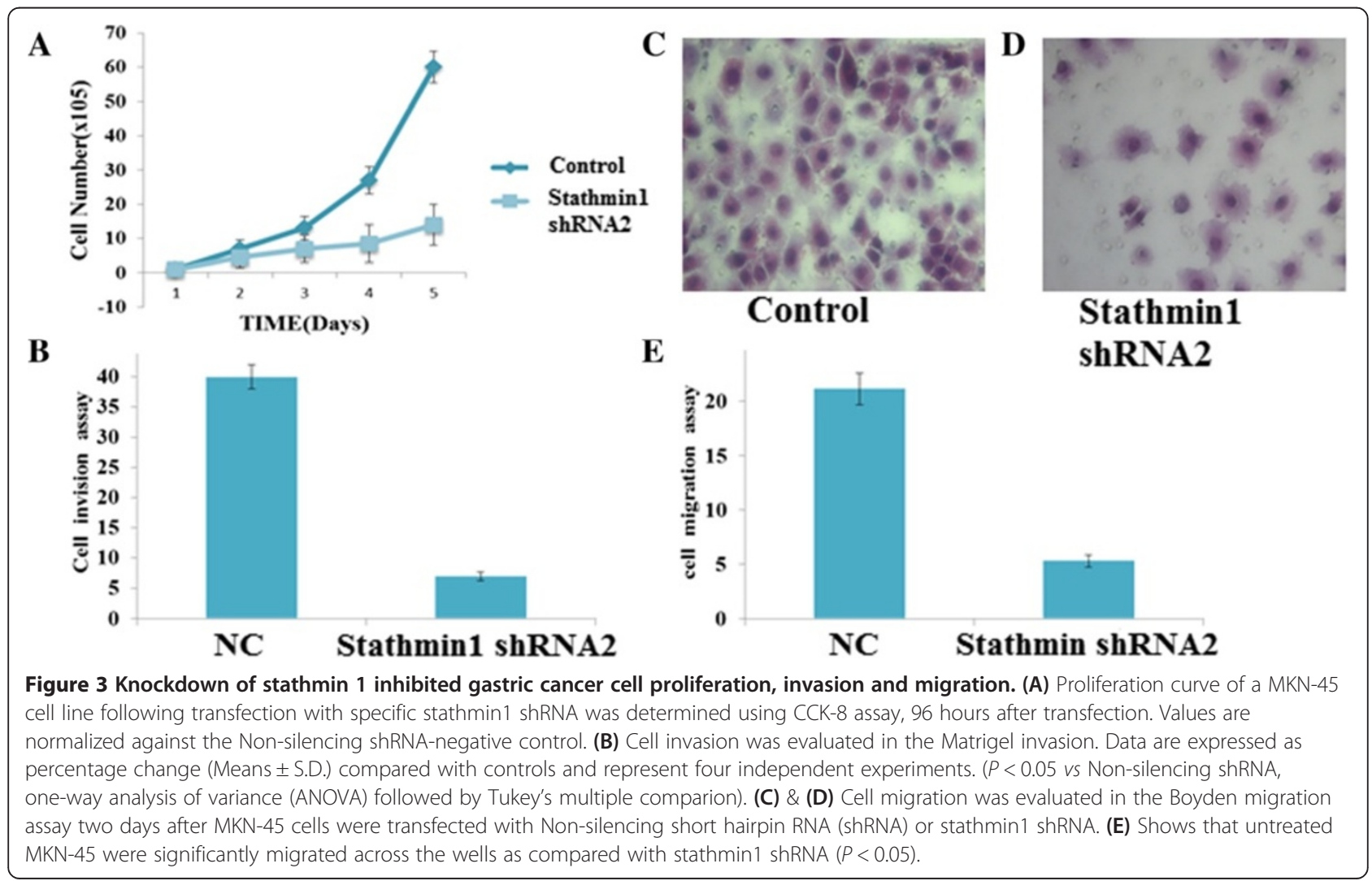



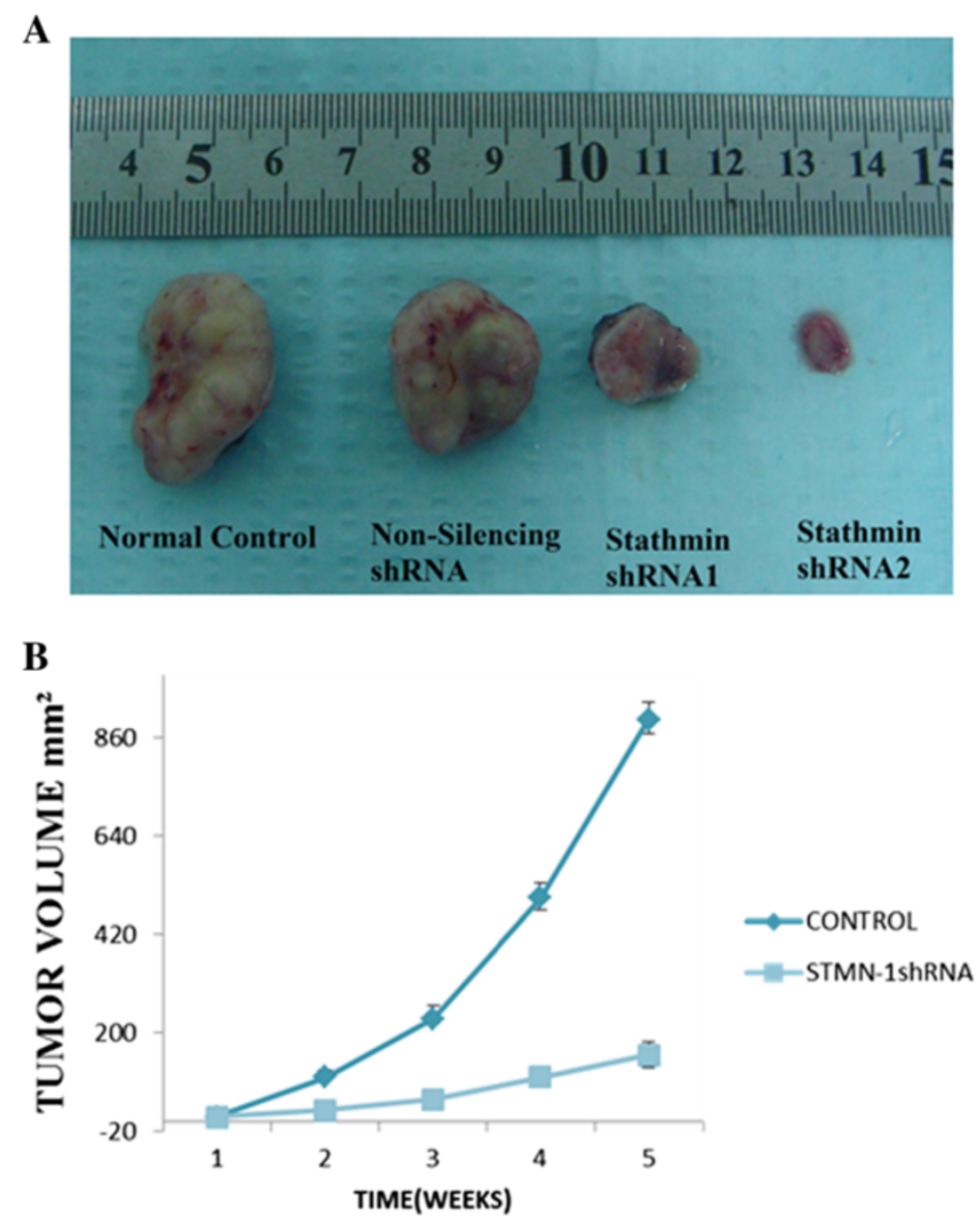

Figure 4 Silencing effect of stathmin shRNA on gastric cancer xenograft in nude mice. MKN-45 cells were transfected with stathmin 1 (STMN1) shRNA or, Non-silencing short hairpin RNA (shRNA) and nude mice were inoculated subcutaneously with $2 \times 10^{6}$ cells at two sites per mouse. The tumor mass (xenograft) volume was measured every week from week 3 to week 7. Data are expressed as the (Means \pm S.D) and represent six independent experiments. ( $P<0.05$ vs Non-silencing shRNA, one-way analysis of variance (ANOVA) followed by Tukey's multiple comparison). (A) Photograph of xenografts dissected from nude mice after 7 weeks subcutaneous inoculation showing suppression growth of cancer cells transfected with stathmin1 shRNA2 rather than non-treated control group. (B) Tumor growth curve showing a significant growth tendency in non-infected group (control) as compared to the stathmin shRNA2 transfected group $(p<0.05)$.

\section{Discussion}

The cell cycle is a tightly regulated process, with each step carefully monitored by a specific group of proteins that act as checkpoints for proper cell division. The balance between these key checkpoints proteins is critical for the progression through each step of the cell cycle to occur. Stathmin 1 (STMN-1), also known as p17, p18, p19, $19 \mathrm{~K}$, metablastin, oncoprotein 18, LAP 18 and $\mathrm{Op} 18$, is a $19 \mathrm{kDa}$ cytosolic protein. Its function as an important regulatory protein of microtubule dynamics has been well characterized [7]. It has been reported that stathmin 1 is overexpressed in many human malignancies, such as leukemia, lymphoma, neuroblastoma, ovarian, prostatic, breast and lung cancers [18] and the modulation of its expression correlates with invasion and metastasis. The protein expression of stathmin 1 has been explored and found to correlate to clinicopathologic factors and poor prognosis in several cancers in different tissues such as brain [19], oral mucosa [20], breast [12,21,22], urothelial [23] as well as ovarian [24], melanoma [25] and uterine cervix [26]. Recently some reports showed that stathmin-1 is related to lymph node metastasis either [27]. Our study on esophageal squamous cell cancer revealed that stathmin 1 is a predictor of survival in stage IIA esophageal squamous cell carcinoma [16].

Stathmin1 has been implicated in G1-S checkpoint control of cell cycle progression by influencing the dynamics of microtubule formation and progression of the cell cycle [28]. In human cancers, Stathmin1 overexpression is associated with increased malignancy, metastasis formation and decreased patient overall survival suggesting that 
Stathmin 1 could serve as a molecular marker to identify patients with more aggressive disease [29]. Recent studies have shown that inhibition of STMN-1 expression in malignant cells interferes with their orderly progression through the cell cycle and abrogates their transformed phenotype [30]. Thus, Stathmin1 provides an attractive molecular target for disrupting the mitotic apparatus and arresting the growth of malignant cells.

In less than a decade after discovery, RNA interferencemediated gene silencing is already being tested as potential therapy in clinical trials for a number of diseases. Lentiviral vectors provide a means to express short hairpin RNA (shRNA) to induce stable and long-term gene silencing in both dividing and non-dividing cells [31]. Lentivirus-mediated RNAi also efficiently knockdown cancer gene expression in lung cancer $[32,33]$ also decreased tumorigenicity in human oral carcinoma cells [34]. RNA interference using small inhibitory RNA (siRNA) has become a powerful tool to downregulate mRNA levels by cellular nucleases that become activated when a sequence homology between the siRNA and a respective mRNA molecule is detected. Therefore siRNA can be used to silence genes involved in the pathogenesis of various diseases associated with a known genetic background. RNAi technology facilitates a posttranscriptional gene silencing via short double-stranded RNA which is capable of binding to a specific mRNA sequence and down regulating the gene expression [35]. However, one of the major barriers to realizing the full medicinal potential of RNAi is the difficulty of delivering it in vivo and the knockdown effect of regular synthesized siRNA only lasts for a short time and does not allow the stable inhibition of target gene function. Lentivirus-mediated delivery of shRNA is more efficient and effective over other systems as it will allow for gene silencing in non-dividing cells [36]. Some studies showed that shRNA are potential therapeutic for gene therapy [37]. RNA interference also provides a paradigm to develop strategies to inactivate essential genes promoting neoplastic growth [38]. Recent advances in gene therapy approaches also indicate that systemic treatment with lentiviral shRNA vectors may be feasible. However, some form of targeted delivery approach may be needed for this. The lentiviral vectors for shRNA expression were used in our system. This approach allows for the stable suppression of target gene expression both in cell culture conditions and in animals. We found that stable knockdown of statmin1 led to reduced proliferation rates (Figure $3 \mathrm{~A}$ ) and migration in vitro (Figure 3C, D) and decreased in vivo tumorigenicity (Figure 4A, B).

Stathmin1 expression in oral cancer found to be correlated with tumor progression and poor prognosis [20]. STMN-1 increases the proliferation rate of leukemia cells [39]. We evaluated the efficacy of stathmin shRNA on the migration and proliferation of gastric cancer cells.
Our findings showed that stathmin shRNA transfection in gastric cancer cells dramatically suppressed proliferation and inhibit migration ability.

To validate effectiveness of down regulation of stathmin1 expression, we performed in vivo studies in nude mice; tumor growth was greatly slowed down in stathmin1 shRNA transfected cells while non-silencing shRNA infected xenograft and control graft grew aggressively in mice model. In future, lentiviral-based RNAi, because of its potency, could be utilized as an effective strategy for cancer therapy. Additionally, the blockage of proliferation in gastric cell lines and the inhibition of tumorigenesis in nude mice support the effectiveness of this strategy. Of the new anticancer drugs approved by the U.S. Food and Drug Administration (FDA) since 2000, fifteen have been targeted drug therapies, compared with only five traditional chemotherapeutic agents [40]. Since the stathmin 1 is critical for growth and the invasive behavior of gastric cancer, silencing of the stathmin 1 gene with RNAi may provide a novel strategy for investigation of the role of stathmin 1 gene in the invasion of human gastric cancers.

\section{Conclusion}

Our findings provide compelling evidence of a novel role for the stathmin1 gene expression in pathogenesis of gastric cancer. We have shown that statmin1 gene expression is crucial for cell proliferation, migration in vitro and tumorigenicity in nude mice. Lentivirusmediated delivery of stathmin1 shRNA has effectively down regulated the expression of stathmin1 gene on mRNA and protein levels. Moreover, silencing of stathmin1 has led to the significant decrease in proliferation and inhibition of migration of MKN-45 cells in vitro and slowdown the tumor growth in nude mice. Therefore, these results suggest that the proliferation and cell migration of MKN-45 cells could be regulated by silencing stathmin 1 gene which is a promising gene therapeutic method to treat gastric cancer.

\section{Competing interests}

The authors declare that they have no competing interests.

\section{Authors' contributions}

JA conceived the study and was involved in its design, performed the RNAi transfection experiments in vitro and in vivo, acquisition of data, statistical analysis and drafted the manuscript. JA and ZPZ performed laboratory experiments, analyzed and interpreted the data. MMB and JA culture the cells and established xenograft tumor model in mice. ZW supervised the study, helped to analyze the data, participated in the statistical analysis, and revising it critically for important intellectual content. All authors read and approved the final manuscript. No writing assistance was used in the production of the manuscript.

Received: 16 July 2013 Accepted: 11 September 2013

Published: 16 September 2013 


\section{References}

1. Jemal A, Bray F, Center MM, Ward E, Ferlay J, Forman D: Global cancer statistics. CA Cancer J Clin 2011, 61:69-90.

2. Parkin D: International variation. Oncogene 2004, 23:6329-6340.

3. Parkin D, Bray F, Devesa S: Cancer burden in the year 2000. The global picture. Eur J Cancer 2001, 37(Suppl 8):S4-S664.

4. Brenner $H$, Rothenbacher D, Arndt V: Epidemiology of stomach cancer. Methods Mol Biol 2009, 472:467-477.

5. Jemal A, Siegel R, Ward E, Murray T, Xu J, Smigal C, Thun M: Cancer statistics. CA Cancer J Clin 2006, 56:106-130.

6. Hudler P: Genetic aspects of gastric cancer instability. ScientificWorldJournal 2012, 2012:761909.

7. Sobel A: Stathmin: a relay phosphoprotein for multiple signal transduction. Trends Biochem Sci 1991, 16:301-305.

8. Doye V, Gouvello SL, Dobransky T: Expression of transfected stathmin cDNA reveals novel phosphorylated forms associated with developmental and functional cell regulation. Biochem J 1992, 287:549-554.

9. Luo X-N, Mookerjee B, Ferrari A, Mistry S, Atweh G: Regulation of phosphoprotein p18 in leukemic cells. J Biol Chem 1994, 269(14):10312-10318.

10. Bieche I, Lachkar S, Becette V: Overexpression of the Stathmin gene in a subset of human breast cancer. Br J Cancer 1998, 78(6):701-709.

11. Jeon $T$, Han $M$, Lee $Y$ : Overexpression of stathmin 1 in the diffuse type of gastric cancer and its roles in proliferation and migration of gastric cancer cells. Br J Cancer 2010, 102:710-718.

12. Brattsand $\mathrm{G}$ : Correlation of oncoprotein $18 /$ stathmin expression in human breast cancer with established prognostic factors. Br J Cancer 2000, 83:311-318.

13. Chen G, Wang H, Gharib T, Huang C, Thomas D, Shedden K, Kuick R, Taylor J, Kardia S, Misek D: Overexpression of oncoprotein 18 correlates with poor differentiation in lung adenocarcinomas. Mo/ Cell Proteomics 2003, 2:107-116.

14. Melhem R, Strahler J, Hailat N, Zhu X, Hanash S: Involvement of OP18 in cell proliferation. Biochem Biophys Res Commun 1991, 179:1649-1655.

15. Friedrich B, Gronberg $H$, Landstrom M, Gullberg M, BergBh A: Differentiation-stage specific expression of oncoprotein 18 in human and rat prostatic adenocarcinoma. Prostate 1995, 27:102-109.

16. Akhtar J, Wang Z, Yu C, Zhang ZP, Bi MM: STMN-1 gene: a predictor of survival in stage IIA esophageal squamous cell carcinoma after IvorLewis esophagectomy? Ann Surg Oncol 2013. Epub ahead of print.

17. Elbashir MS, Lendeckel W, Tuschl T: RNA interference is mediated by 21and 22-nucleotide RNAs. Genes Dev 2001, 15:188-200.

18. Rana S, Maples PB, Senzer N, Nemunaitis J: Stathmin 1: a novel therapeutic target for anticancer activity. Expert Rev Anticancer Ther 2008, 8:1461-1470.

19. Kuo M-F, Wang H-S, Kuo Q-T, Shun C-T, Hsu H-C, Yang S-H, Yuan R-H: High expression of stathmin protein predicts a fulminant course in medulloblastoma. J Neurosurg Pediatr 2009, 4:74-80.

20. Kouzu Y, Uzawa K, Koike H, Saito K, Nakashima D, Higo M, Endo Y, Kasamatsu A, Shiiba M, Bukawa $H$, et al: Overexpression of stathmin in oral squamous-cell carcinoma:correlation with tumour progression and poor prognosis. Br J Cancer 2006, 94(5):717-723.

21. Saal LH, Johansson P, Holm K, Gruvberger-Saal SK, She Q-B, Maurer M, Koujak S, Ferrando AA, Malmström P, Memeo L, et al: Poor prognosis in carcinoma is associated with a gene expression signature of aberrant PTEN tumor suppressor pathway activity. Proc Natl Acad Sci USA 2007, 1(104):7564-7569.

22. Golouh R, Cufer T, Sadikov A, Nussdorfer P, Usher PA, Brünner N, Schmitt M, Lesche R, Maier S, Timmermans M, et al: The prognostic value of Stathmin-1, S100A2, and SYK proteins in ER-positive primary breast cancer patients treated with adjuvant tamoxifen monotherapy: an immunohistochemical study. Breast Cancer Res Treat 2008, 110:317-326.

23. Lin W-C, Chen S-C, Hu F-C, Chueh S-C, Pu Y-S, Yu H-J, Huang K-H: Expression of stathmin in localized upper urinary tract urothelial carcinoma: correlations with prognosis. Urology 2009, 74:1264-1269.

24. Su D, Smith SM, Preti M, Schwartz P, Rutherford TJ, Menato G, Danese S, Ma S, Yu H, Katsaros D: Stathmin and tubulin expression and survival of ovarian cancer patients receiving platinum treatment with and without paclitaxel. Cancer 2009, 1(115):2453-2463.

25. Chen J, Abi-Daoud M, Wang A, Yang X, Zhang X, Feilotter H, Tron V: Stathmin 1 is a potential novel oncogene in melanoma. Oncogene 2012, 10:1038

26. Xi W, Rui W, Fang L, Ke D, Ping G, Hui-Zhong Z: Expression of stathmin/ op18 as a significant prognostic factor for cervical carcinoma patients. J Cancer Res Clin Oncol 2009, 135:837-846.
27. Trovik J, Wik E, Stefansson IM, Marcickiewicz J, Tingulstad S, Staff AC, Njolstad TS, MoMaTec Study Group, Vandenput I, Amant F, et al: Stathmin overexpression identifies high-risk patients and lymph node metastasis in endometrial cancer. Clin Cancer Res 2011, 17:3368-3377.

28. Sherbet GV, Cajone F: Stathmin in cell proliferation and cancer progression. Cancer Genomics \& Proteomics 2005, 2:227-238.

29. Belletti B, Baldassarre G: Stathmin: a protein with many tasks. New biomarker and potential target in cancer. Expert Opin Ther Targets 2011, 15:1249-1266

30. Mistry S, Atweh $\mathrm{G}$ : Role of stathmin in the regulation of the mitotic spindle: potential applications in cancer therapy. Mt Sinai J Med 2002, 69:299-304.

31. Manjunath $\mathrm{N}, \mathrm{Wu} \mathrm{H}$, Subramanya S, Shankar P: Lentiviral delivery of short hairpin RNAs. Adv Drug Deliv Rev 2009, 61:732-745.

32. Guo X, Wang W, Hu J, Feng K, Pan Y, Zhang L, Feng Y: Lentivirus-mediated RNAi knockdown of NUPR1 inhibits human nonsmall cell lung cancer growth in vitro and in vivo. Anat Rec (Hoboken) 2012, 295:2114-2121.

33. Feng Y, Hu J, Ma J, Feng K, Zhang X, Yang S, Wang W, Zhang J, Zhang Y: RNAi-mediated silencing of VEGF-C inhibits non-small cell lung cancer progression by simultaneously down-regulating the CXCR4, CCR7, VEGFR-2 and VEGFR-3-dependent axes-induced ERK, p38 and AKT signalling pathways. Eur J Cancer 2011, 47:2353-2363.

34. Pozzi V, Sartini D, Morganti S, Giuliante R, Ruscio GD, Santarelli A, Rocchetti R, Rubini C, Tomasetti M, Giannatempo G, et al: RNA-mediated gene silencing of nicotinamide N-Methyltransferase is associated with decreased tumorigenicity in human oral carcinoma cells. PLoS One 2013, 8:e71272.

35. Abbas-Terki T, Blanco-Bose W, Déglon N, Pralong W, Aebischer P: Lentiviralmediated RNA interference. Hum Gene Ther 2002, 13:2197-2201.

36. Stewart SA, Dykxhoorn DM, Palliser D, Mizuno H, Yu EY, An DS, Sabatini DM, Chen ISY, Hahn WC, Sharp PA, et al: Lentivirus-delivered stable gene silencing by RNAi in primary cells. RNA 2003, 9:493-501.

37. Koutsilieri E, Rethwilm A, Scheller C: The therapeutic potential of siRNA in gene therapy of neurodegenerative disorder. J Neural Transm 2007, 72:43-49. 43-49.

38. Gaither A, lourgenko V: RNA interference technologies and their use in cancer research. Curr Opin Oncol 2007, 19:50-54.

39. Jeha S, Luo X-N, Beran M, Kantarjian H, Atweh GF: Antisense RNA inhibition of phosphoprotein p18 expression abrogates the transformed phenotype of leukemic cells. Cancer Res 1996, 56:1445-1450.

40. Centerwatch: Drugs approved by the FDA. 2007. http://www.centerwatch. com/patient/drugs/druglist.html. Accessed May 8, 2007.

\section{doi:10.1186/1479-5876-11-212}

Cite this article as: Akhtar et al:: Lentiviral-mediated RNA interference targeting stathmin 1 gene in human gastric cancer cells inhibits proliferation in vitro and tumor growth in vivo. Journal of Translational Medicine 2013 11:212

\section{Submit your next manuscript to BioMed Central and take full advantage of:}

- Convenient online submission

- Thorough peer review

- No space constraints or color figure charges

- Immediate publication on acceptance

- Inclusion in PubMed, CAS, Scopus and Google Scholar

- Research which is freely available for redistribution 